\title{
Generation Process and Analysis of Innovative Timberfabric Vaults
}

Etienne Albenque, Master's student; Markus Hudert, Dr; Laurent Humbert, Dr; Yves Weinand, Prof. Dr; Laboratory for Timber Constructions (IBOIS), Ecole Polytechnique Fédérale de Lausanne (EPFL), Lausanne, Switzerland. Contact: yves.weinand@epfl.ch

DOI: $10.2749 / 101686614 X 13830790993285$

\begin{abstract}
Timberfabric structures, developed at IBOIS, are a new family of timber constructions based on the logic and principles of man-made fabrics. Modular elements called "timberfabric modules" are generated by curving and connecting two thin wooden panels. This paper concerns the study of vaults obtained by assembling together several timberfabric modules. A parametric tool is presented which can generate automatically a three-dimensional (3D) finite element model of a structure for a given set of initial parameters. This tool assists the architectural design of such structures, providing insights into their geometry as well as into the construction stresses and the kinematic constraints between different constitutive elements.
\end{abstract}

Keywords: timberfabric vaults; innovative structures; Python script; generative algorithm; assembly process.

\section{Introduction}

In recent years, textiles have increasingly served as reference for both architects and civil engineers. ${ }^{1,2}$ The research project Timberfabric ${ }^{3,4}$ concentrates especially on the assembly principles and techniques that are used for the production of textile structures. By combining these principles with the particular properties of laminated timber panels, the work sets out to develop a modular construction system for supporting building envelopes. During the first stage of the research, the basic unit for this system was developed. This basic unit, which in the following is referred to as timberfabric module, consists of two interlaced, mutually supporting timber panels. $^{5,6}$ Based on this unit, a considerable amount of empirical studies has been carried out in which various vaultshaped, multi-module configurations were developed. ${ }^{7}$ In parallel, numerical models have been developed to generate the deformed shape of several particular configurations of one timberfabric module. ${ }^{8}$ Figure 1 shows a prototype of a vault built by connecting together full timberfabric modules and halves of timberfabric modules. The connectors are made out of planar

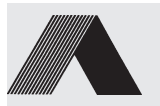

Peer-reviewed by international experts and accepted for publication by SEI Editorial Board

Paper received: March 28, 2013

Paper accepted: June 27, 2013 wooden panels cut by a numerically controlled machine. The design process of this prototype is based on an empirical approach, the principal steps of which are summarized by a simplified diagram (Fig. 2).

The complexity of the structural and architectural design of a timberfabric vault comes from the fact that its spatial configuration depends on the geometry of the timberfabric module which in turn depends on the geometry, material properties and assembly conditions of the timber panels. Moreover, construction stresses are generated during the assembly of the timberfabric modules. Those stresses will affect the resistance and the behavior of the finished structure.

The objective of the work presented in this paper was to develop a parametric tool to assist the structural and architectural design of the timberfabric vault. The research presented in this paper is focused on one particular family of timberfabric vaults. However it aims at establishing an analytical framework for all Timberfabric structures and describes the current state of a numeric tool that can be developed further.

The developed tool permits the automatic generation of a three-dimensional (3D) finite element (FE) model of a timberfabric vault for a given set of initial parameters. This model can be used to study not only the spatial quality but also the structural behavior and the resistance of the projected structure. The elaboration of this tool is presented in three steps.

Firstly, the strategy adopted to generate a 3D FE model of one timberfabric module is presented, with given boundary conditions, by simulating the deformation of two flat wooden panels for which the user can specify the dimensions and the mechanical properties.

Secondly, several geometric properties shared by the resulting timberfabric modules are highlighted and "intermediate variables" are introduced to describe the geometry obtained for the given set of initial parameters. Also the results of a parametric study show the influence of the initial parameters of the flat wooden panels on the intermediate variables that describe the geometry of the corresponding timberfabric module.

Finally, it is shown how those intermediate variables can be used to describe the relative position of timberfabric modules in a timberfabric vault system. The numeric tool of the first part is therefore completed in order to enable the automatic generation of a full vault from one timberfabric module. The geometry of the connection pieces and their behavior are also generated automatically.

The different steps presented in this paper do not follow the same logic of the empirical approach developed before. In contrast, several initial hypotheses are based on observations of the built prototypes and their relevance to the modeling of a physical reality is demonstrated in the core of the paper. This chronology illustrates the complementarities between empirical and numerical approaches to design.

\section{Parametric Model for the Generation of a Timberfabric Module}

This section describes the routine employed to generate the geometry 


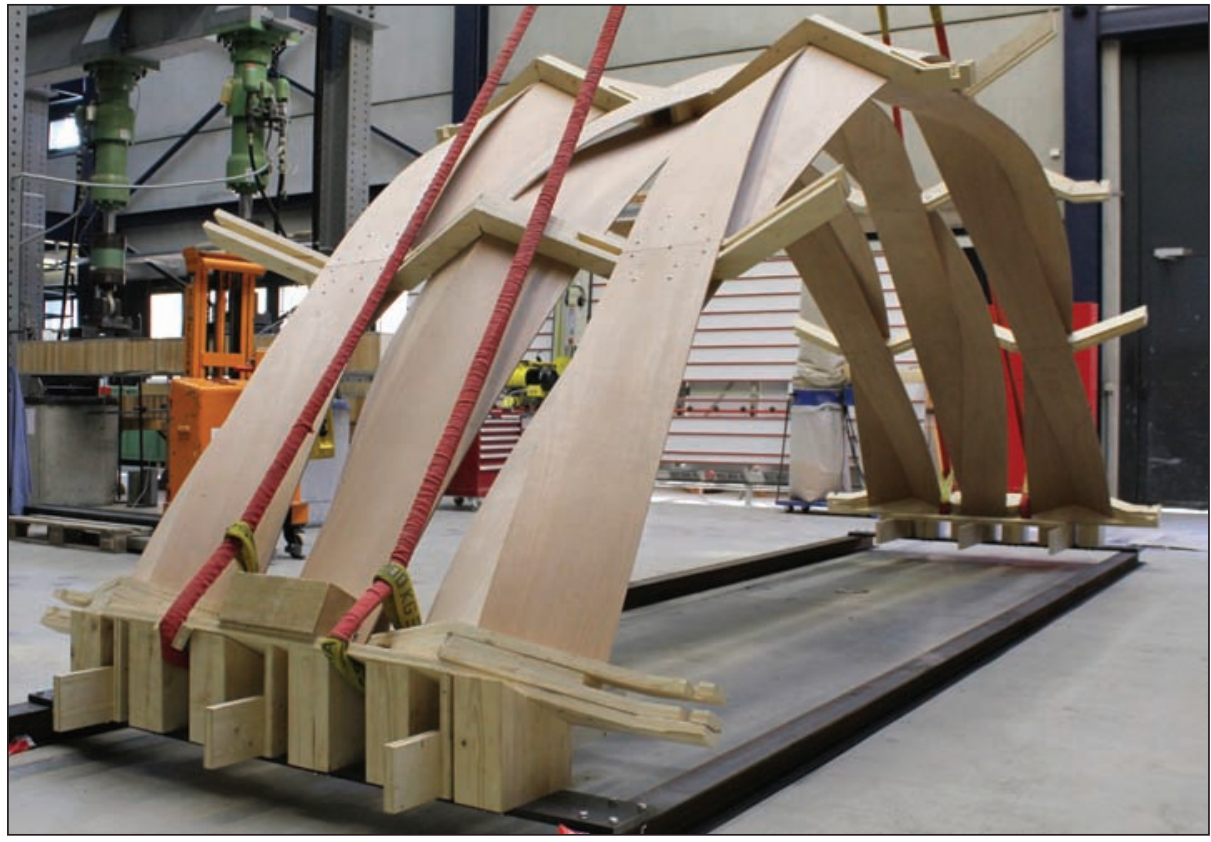

Fig. 1: Timberfabric vault prototype

and calculate the initial stress state of a timberfabric module for a given set of geometric parameters and material properties of the timber panels. It is based on an editable script, written under the FE scripting interface environment ${ }^{9}$ that builds and runs a nonlinear FE model.

\section{Geometrical Parameters and Material Properties of the Panels}

Timberfabric modules (TMs) can be regarded as elementary unit cells that are connected together to form a more complex spatial structure such as the vault prototype depicted in Fig. 1. The module is formed by two self-supporting, initially flat, rectangular wooden panels that are deformed and assembled together by attachment at their extremities. The two panels of a module come into contact at a single point on one of their longitudinal edges. The width, the length and the thickness of the undeformed planar panels are noted respectively as $W, L$ and $t$ (see Fig. 2).

Depending on the scale of the timberfabric module, the timber panels are either made out of plywood panels (for reduced-scale prototype) or laminated wood panels (for full-scale TMs). In both cases, the panels are composed of orthotropic layers that are considered to be rigidly bonded together. The behavior of a panel at a macroscopic scale is viewed as equivalent to the behavior of a single-layer, homogeneous, orthotropic material with principal (material) axes along the longitudinal and transversal geometrical directions of the panel. With those assumptions and considering a linear elastic constitutive law, only four independent constants are required to fully characterize the material behavior: the Young's moduli in the two principal directions $E_{1}$ and $E_{2}$, the in-plane shear modulus $G_{12}$ and the Poisson's ratio $v_{12} \cdot{ }^{10,11}$ Moisture content and time dependance of the material properties are not taken into account.

\section{Analysis Framework}

The FE code is used to build a 3D model of the deformation process that enables one to generate a timberfabric module from two planar timber panels. Rather than using the graphical user interface, the FE model is generated from the instructions written in a script using the programming language Python. ${ }^{9}$ By automating the construction of the FE model, this method allows one to vary parameters very easily.

The timber panels are meshed using four-node, quadrilateral shell elements $\mathrm{S} 4 \mathrm{R}$ with reduced integration and six active degrees of freedom per node These general-purpose elements are suitable for the analysis of (doubly) curved thick or thin shells, allowing transverse shear deformation. Because large rotations and displacements are expected, a geometrically nonlinear analysis is conducted for which the imposed displacements are applied incrementally. 12

The geometry of the panels is described by reference to their middle surface. The position vector of a point/node $M$ of the middle surface is noted $\mathbf{r}^{M}$ and the unit vector normal to the middle surface at this point is denoted by $\hat{\mathbf{n}}^{M}$ Moreover, $\mathbf{u}^{M}$ and $\boldsymbol{\theta}^{M}$ are respectively the displacement and rotation vectors at $M$ with components $u_{j}^{M}$ and $\theta_{j}^{M}$ given along a base vector $\hat{\mathbf{e}}_{j}$. In particular, $\theta_{j}^{M}$ is the component of the rotation of the normal vector around the base vector $\hat{\mathbf{e}}_{j}$.
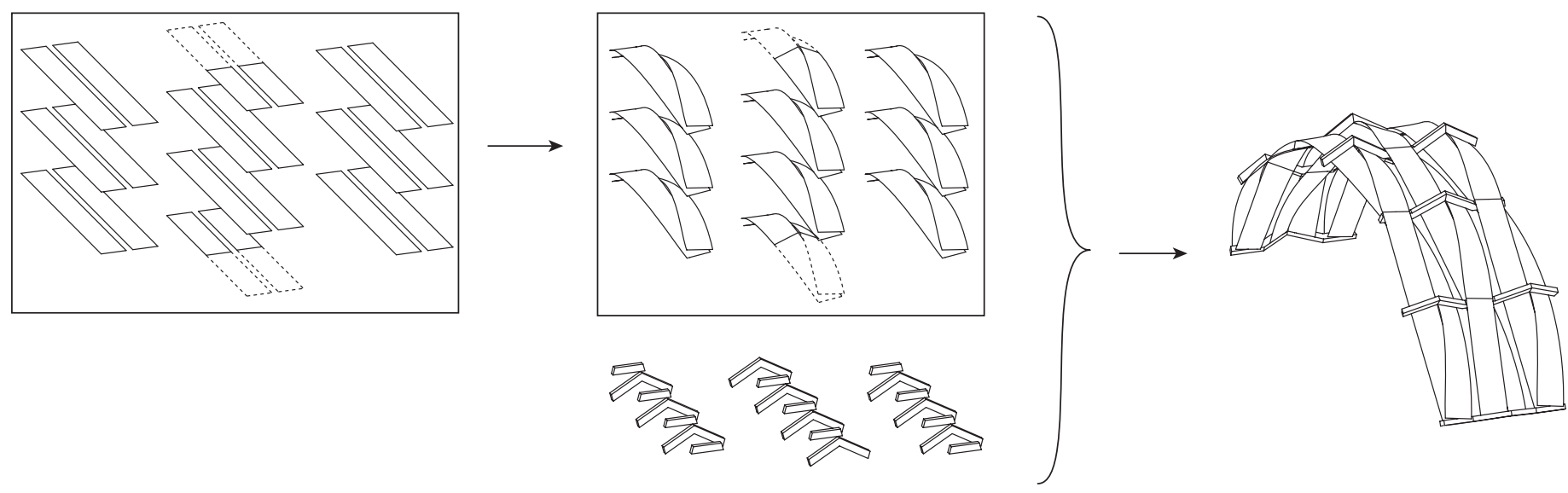

Fig. 2: Construction process of timberfabric vaults 
(a)

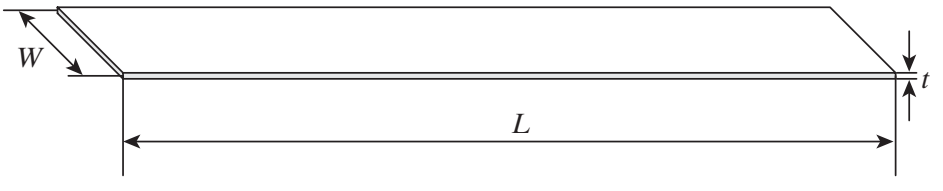

(b)

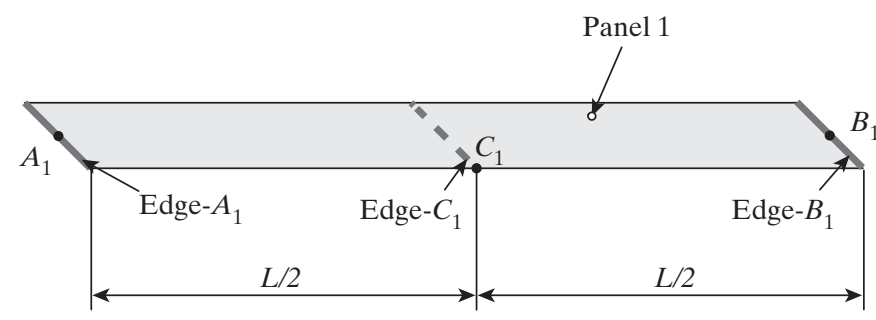

(c)

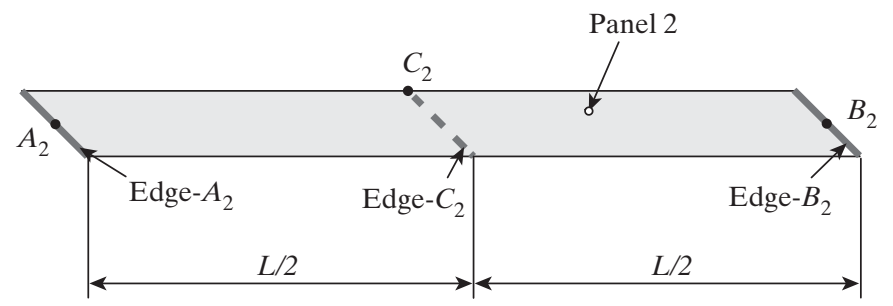

Fig. 3: (a) Panels parameterization. (b, c) mid surface of panels 1 and 2, definition of geometric entities

In the following, the same annotation will be used for node sets and for the corresponding geometric points. Depending on the context, the superscript $M$ will either refer to a node or to the geometric point of coordinates $\mathbf{r}^{M}=\left(r_{1}^{M}, r_{2}^{M}, r_{3}^{M}\right)^{T}$.

Let $A_{1}, B_{1}, A_{2}$, and $B_{2}$, be the midpoints of the transversal edges of panels (1) and (2) as represented in Fig. 3. The mid-points of a longitudinal edge of panels (1) and (2) are $C_{1}$ and $C_{2}$ and Edge- $A_{1}$, Edge- $B_{1}$, Edge- $A_{2}$, Edge$B_{2}$, Edge- $C_{1}$, and Edge- $C_{2}$, refer to the transversal edges corresponding to the points $A_{i}, B_{i}$ and $C_{i}$. Once again, the same notation is used for the edge as a geometric entity and the edge as a set of node numbers.

\section{Initial Position and Boundary \\ Conditions}

\section{Initial Position}

Let $\left(\mathbf{O}, \hat{\mathbf{e}}_{1}, \hat{\mathbf{e}}_{2}, \hat{\mathbf{e}}_{3}\right)$ be an orthonormal cartesian coordinate system. Initially, the panels are positionned in the $\left(x_{1}, x_{2}\right)$ plane with their longitudinal axes along $\hat{\mathbf{e}}_{1}$. For each panel, the initial position of the nodes introduced previously are such that $\mathbf{r}^{A_{1}}=\mathbf{r}^{A_{2}}=\mathbf{O}, \mathbf{r}^{B_{1}}=\mathbf{r}^{B_{2}}=(L, 0,0)^{T}, \mathbf{r}^{C_{1}}=$ $\left(\frac{L}{2},-\frac{W}{2}, 0\right)^{T}, \mathbf{r}^{C_{2}}=\left(\frac{L}{2}, \frac{W}{2}, 0\right)^{T}$.

In the subsequent text, $\Delta(M, \hat{\mathbf{n}})$ will stand for the straight line through a point $M$, directed by a (unit) vector $\hat{\mathbf{n}}$ and $\Pi(M, \hat{\mathbf{n}})$ for the plane of normal $\hat{\mathbf{n}}$ at $M$.

\section{Initial Boundary Conditions}

The following boundary conditions are imposed to block certain displacement degrees of freedom of the nodes $A_{1}$, $A_{2}, B_{1}$ and $B_{2}$ :

- $A_{1}$ is fixed: $u_{1}^{A_{1}}=u_{2}^{A_{1}}=u_{3}^{A_{1}}=0$

- $B_{2}$ is constrained on line : $\Delta\left(A_{1}, \hat{\mathbf{e}}_{2}\right)$ : $u_{2}^{B_{2}}=u_{3}^{B_{2}}=0$

- $A_{2}$ and $B_{1}$ are constrained in plane : $\prod\left(A_{1}, \hat{\mathbf{e}}_{2}\right): u_{2}^{A_{2}}=u_{2}^{B_{1}}=0$

\section{Interactions and Imposed Displacements}

\section{Interaction Conditions}

In the actual process of fabrication, the panels are deformed in several sequential steps with intervening temporary means of fixation. During the last step, the edges of the panels are forced into position in order to adapt to certain geometric conditions imposed by connection pieces. For the numerical simulation, the authors chose to impose some of those conditions beforehand.

In the FE model, imposing interaction conditions consists in eliminating degrees of freedom (DOF) of a group of nodes and coupling their motion to that of a single node (called the "master node" in the FE program). For example, if all the displacement degrees of freedom of the nodes of a straight edge are coupled to the displacement DOFs of one node of the edge, then the nodes will be aligned for any deformation state of the sys- tem, simulating therefore the effect of a straight rigid piece of connection pinned continuously along the edge.

The following interacting conditions impose kinematic constraints respectively between the degrees of freedom of the node sets Edge- $A_{1}$, Edge- $B_{1}$, Edge- $C_{1}$, Edge- $A_{2}$, Edge- $B_{2}$, Edge$C_{2}$ and the nodes $A_{1}, B_{1}, C_{1}, A_{2}, B_{2}, C_{2}$. To simplify the expressions, these constraints are written using the position and normal vector of the nodes.

The first set of constraints forces the panels to be tangential to planes along their extreme transversal edges (Edge- $A_{1}$, Edge- $B_{1}$, Edge- $A_{2}$, Edge$B_{2}$ ) while at each extremity of the timberfabric module, the planes for each panel are constrained to be parallel (they have the same normal vector):

- Panel 1 has to remain tangential to the plane $\Pi\left(A_{1}, \hat{\mathbf{n}}^{A_{1}}\right)$ along Edge- $A_{1}$ and to the plane $\Pi\left(B_{1}, \hat{\mathbf{n}}^{B_{1}}\right)$ along Edge- $B_{1}$ :

$$
\begin{aligned}
& \left(\mathbf{r}^{M}-\mathbf{r}^{A_{1}}\right) \cdot \hat{\mathbf{n}}^{A_{1}}=0, \hat{\mathbf{n}}^{M} \times \hat{\mathbf{n}}^{A_{1}}=0, \\
& M \in E d g e-A_{1} \\
& \left(\mathbf{r}^{M}-\mathbf{r}^{B_{1}}\right) \cdot \hat{\mathbf{n}}^{B_{1}}=0, \hat{\mathbf{n}}^{M} \times \hat{\mathbf{n}}^{B_{1}}=0, \\
& M \in E d g e-B_{1}
\end{aligned}
$$

- Panel 2 has to remain tangential to the plane $\Pi\left(A_{2}, \hat{\mathbf{n}}^{A_{1}}\right)$ along Edge- $A_{2}$ and to the plane $\Pi\left(B_{2}, \hat{\mathbf{n}}^{B_{1}}\right)$ along Edge $-B_{2}$ :

$$
\begin{aligned}
& \left(\mathbf{r}^{M}-\mathbf{r}^{A_{2}}\right) \cdot \hat{\mathbf{n}}^{A_{1}}=0, \hat{\mathbf{n}}^{M} \times \hat{\mathbf{n}}^{A_{1}}=0, i \\
& M \in E d g e-A_{2} \\
& \left(\mathbf{r}^{M}-\mathbf{r}^{B_{2}}\right) \cdot \hat{\mathbf{n}}^{B_{1}}=0, \hat{\mathbf{n}}^{M} \times \hat{\mathbf{n}}^{B_{1}}=0, \\
& M \in E d g e-B_{2}
\end{aligned}
$$

The second set of constraints is imposed on the central transversal edges of the panels Edge- $C_{1}$, Edge- $C_{2}$. The nodes of those edges are to remain aligned:

$$
\begin{aligned}
& \left(\mathbf{r}^{M}-\mathbf{r}^{C_{1}}\right) \times\left(\mathbf{r}^{M^{\prime}}-\mathbf{r}^{C_{1}}\right)=0, \\
& M, M^{\prime} \in E d g e-C_{1} \\
& \left(\mathbf{r}^{M}-\mathbf{r}^{C_{2}}\right) \times\left(\mathbf{r}^{M^{\prime}}-\mathbf{r}^{C_{2}}\right)=0, \\
& M, M^{\prime} \in E d g e-C_{2}
\end{aligned}
$$

\section{Imposed Displacements}

To simulate the assembly of a timberfabric module, displacements are imposed in three consecutive steps at nodes $A_{2}, B_{1}, C_{1}$ and $C_{2}$ :

In the first step, the displacement of node $A_{2}$ is constrained along the straight line $\Delta\left(A_{1}, \hat{\mathbf{n}}^{A_{1}}\right)$ and the displacement of node $B_{1}$ is constrained along the straight line $\Delta\left(B_{2}, \hat{\mathbf{n}}^{B_{2}}\right)$. The norm of those dispacements, noted as $h_{c}$, is also an initial parameter of the design. It specifies the height of the connection 
pieces at the extremities of the TM. Thus, $\mathbf{u}^{A_{2}}=h_{c} \hat{\mathbf{n}}^{A_{1}}$ and $\mathbf{u}^{B_{1}}=h_{c} \hat{\mathbf{n}}^{B_{2}}$.

In Step 2, the authors impose $\mathbf{u}^{C_{1}}=$ $\frac{W}{2} \hat{\mathbf{e}}_{2}+\frac{W}{2} \hat{\mathbf{e}}_{3}$ and $\mathbf{u}^{C_{2}}=-\frac{W}{2} \hat{\mathbf{e}}_{2}+\frac{W}{2} \hat{\mathbf{e}}_{3}$

The imposed displacements on nodes $A_{2}$ and $B_{1}$ are maintained.

The displacements $(W / 2) \hat{\mathbf{e}}_{2}$ and $-(W / 2)$ $\hat{\mathbf{e}}_{2}$ move respectively the points $C_{1}$ and $C_{2}$ in the plane $\Pi\left(A_{1}, \hat{\mathbf{e}}_{2}\right)$, a plane that already contains the points $A_{1}, A_{2}, B_{1}$ and $B_{2}$.

In both cases, the last term $(W / 2) \hat{\mathbf{e}}_{3}$ is added to force adoption of one of the two equiprobable buckling modes of the panels: indeed, the displacement

\begin{tabular}{|c|c|c|c|}
\hline Thickness $\boldsymbol{t}(\mathbf{m m})$ & Width $\boldsymbol{W}(\mathbf{m m})$ & Length $\boldsymbol{L}(\mathbf{m m})$ & Connector height $\boldsymbol{h}_{\boldsymbol{c}}(\mathbf{m m})$ \\
\hline 8,0 & 280 & 2450 & 80 \\
\hline $\left.\boldsymbol{E}_{\mathbf{1}} \mathbf{M P a}\right)$ & $\left.\boldsymbol{E}_{\mathbf{2}} \mathbf{M P a}\right)$ & $v_{\mathbf{1 2}}$ & $\left.\boldsymbol{G}_{\mathbf{1 2}} \mathbf{M P a}\right)$ \\
\hline 4163 & 5088 & 0,3 & 552 \\
\hline
\end{tabular}

Table 1: Geometric and mechanical properties of the panels

along $\hat{\mathbf{e}}_{2}$ induces an important bending moment in the panels along $\hat{\mathbf{e}}_{3}$ and if no displacement along $\hat{\mathbf{e}}_{3}$ was specified, the panels would continue to have two equiprobable lateral buckling modes. For reasons of convergence of the algorithm, the norm of this displacement has the same magnitude order as the expected displacement.

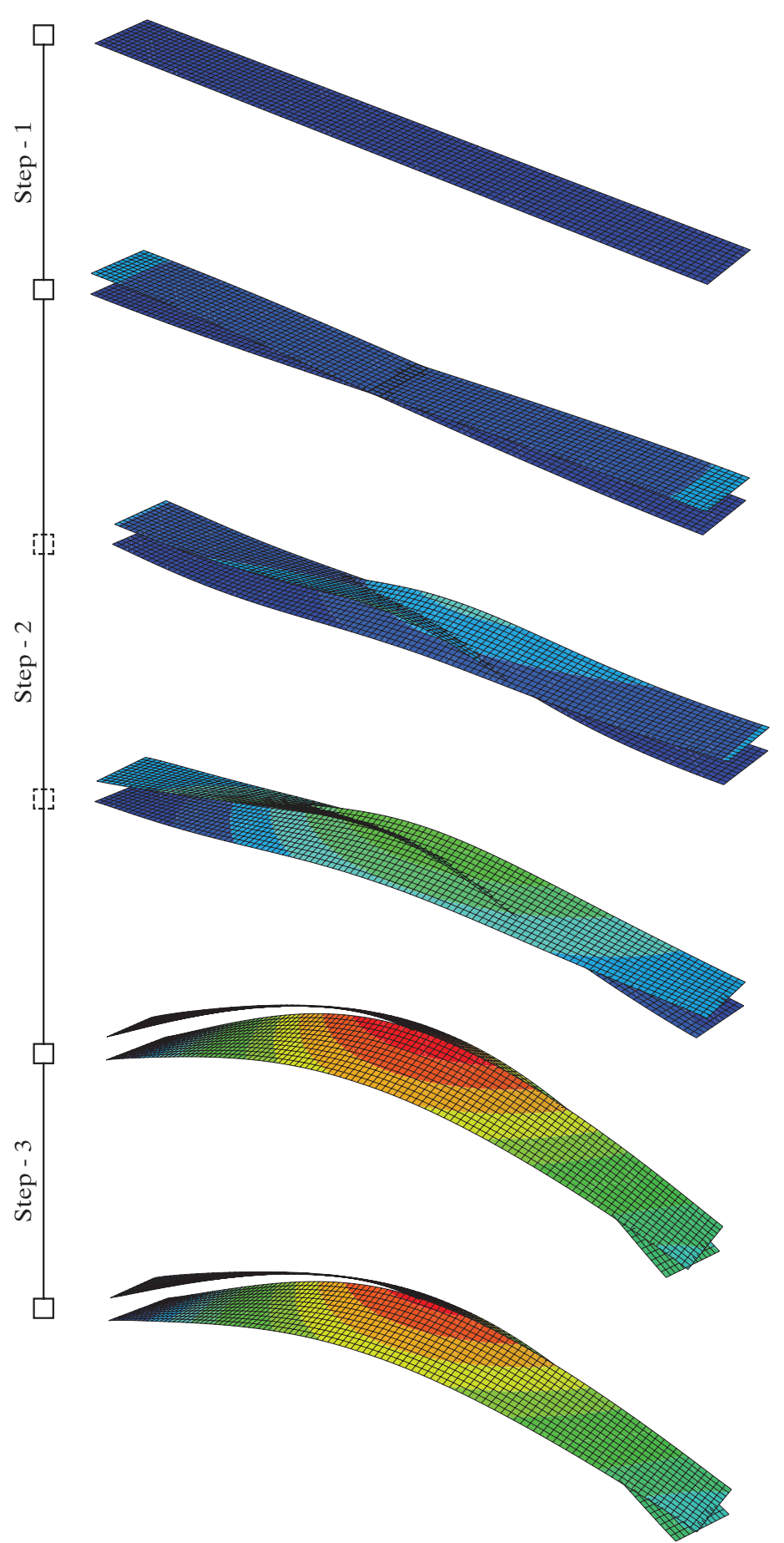

Fig. 4: Calculation steps of the generation process of a timberfabric module
In the last step, the constraints on Edge $-C_{1}$ and Edge $-C_{2}$ are released and the points $C_{1}$ and $C_{2}$ reach their 'natural' post-buckling position.

\section{Example}

For the prototype of the vault shown in the introduction, the panels are made of a commercial Okoumé plywood with characteristic values $E_{1}=4163 \mathrm{MPa}, E_{2}=5088 \mathrm{MPa}$ for the Young's moduli (in traction/compression) and $G_{12}=552 \mathrm{MPa}$ for the in-plane shear modulus. ${ }^{13}$ For convenience, the geometrical and material properties of the panels are reported in Table 1. The deformed shape and displacement fields are reproduced in Fig. 4 at the beginning and at the end of each step of imposed displacements.

The objective of this modeling is to calculate the geometry and the stress state of the timber panels that constitute a TM but is not intended to simulate the real process of fabrication. The authors can thus allow the pair of panels modeled to interpenetrate each other during the calculation and propose a virtual assembly process much simpler than the actual fabrication assembly process of a TM prototype.

\section{Internal Forces and Boundary Reactions in Deformed State}

The process described above allows one to generate the deformed shape of two wooden panels with complex boundary and interaction conditions. At this stage, it is legitimate to wonder if those particular conditions are relevant to describing the physical reality of a timberfabric module.

If there is a boundary condition on node $M$, vector $\mathbf{R}^{M}$ designates the reaction force at the node. In its final deformed shape, the system constituted of the two wooden panels is subjected to several internal forces and to six reaction forces at points $A_{1}, A_{2}$, $B_{1}, B_{2}, C_{1}$, and $C_{2}$. By considering the nature of the boundary conditions and the absence of exterior actions, it can be demonstrated easily that all those forces are colinear to $\mathbf{e}_{2}$ and $\mathbf{R}^{M} \times \hat{\mathbf{e}}_{2}=$ 0 for $M \in\left\{A_{1}, A_{2}, B_{1}, B_{2}, C_{1}, C_{2}\right\}$. 


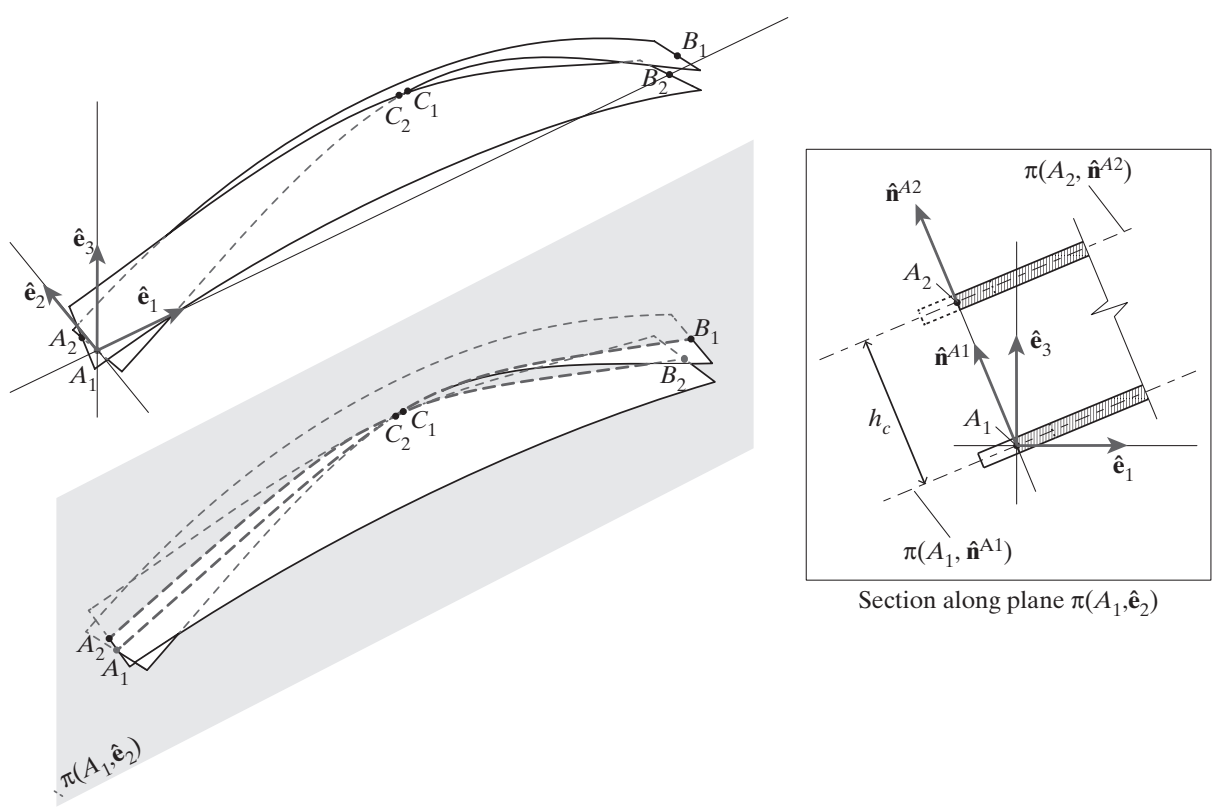

Fig. 5: Geometric characteristics of timberfabric modules

Furthermore, if $C$ is introduced, the point such that $\mathbf{r}^{c}=\left(\mathbf{r}^{c_{1}}+\mathbf{r}^{c_{2}}\right) / 2$, the deformed shape of the panels has an axial symmetry of axis $\Delta\left(C, \hat{e}_{3}\right)$. By using the axial symmetry and the absence of external forces, it can be shown that $\mathbf{R}^{A_{1}}=-\mathbf{R}^{B_{2}}, \mathbf{R}^{A_{2}}=-\mathbf{R}^{B_{1}}$ and $\mathbf{R}^{C_{1}}=-\mathbf{R}^{C_{2}}$.

By observing the numerical results, it is possible to assimilate the reaction $\mathbf{R}^{C_{1}}$ and $\mathbf{R}^{C_{2}}$ forces to internal forces. Numerically, it is observed that the distance between the positions of $C_{1}$ and $C_{2}$ in the deformed state is inferior to $0,5 \%$ of the length of the panels. Accordingly, $\delta=\left\|\mathbf{r}^{C_{2}}-\mathbf{r}^{C_{1}}\right\|<0,005 \cdot L$.

By assuming that this distance is neglectable, the two opposed reaction forces can be assimilated to a contact force between the panels. In order to confirm this hypothesis, the model was run again by slightly shifting the initial position of the points $C_{1}$ and $C_{2}$ such that initially $\mathbf{r}^{C_{1}}=\left(\frac{L+\delta}{2},-\frac{W}{2}, 0\right)^{T}, \mathbf{r}^{C_{2}}=\left(\frac{L-\delta}{2}, \frac{W}{2}, 0\right)^{T}$.

After this iteration, it is observed: $\delta^{\prime}=\left\|\mathbf{r}^{C_{2}}-\mathbf{r}^{C_{1}}\right\|<0,001 \cdot L$. The result of the first round of modeling has thus

Furthermore in next section, several variables will be introduced to quantitatively describe the model. The numerical results of those variables for the two situations described above is negligible, meaning that the first approximaton is acceptable. Finally, the four remaining reactions are consequences of the choice to impose the coplanarity of the points $A_{1}, A_{2}, B_{1}$, and $B_{2}$ : it will be demonstrated in section "Parametric Model for Timber Vaults" that this geometric condition is necessary for the assembly of several timberfabric modules and that the corresponding reaction forces will be equilibrated over one timberfabric module to another.

\section{Geometrical Aspects of Timberfabric Modules}

In order to describe the relative positions of several timberfabric modules that form a timberfabric vault, it is essential to introduce new variables that describe the geometry of a timberfabric module. The definition of such intermediate variables necessitates some geometric observations of the timberfabric modules.

\section{Geometric Description}

Figure 5 illustrates some geometric consequences of the boundary and interaction conditions described in previous section:

- The points $A_{1}, A_{2}, B_{1}, B_{2}, C_{1}$, and $C_{2}$ belong to the plane $\Pi\left(A_{1}, \hat{\mathbf{e}}_{2}\right)$.

- The panels 1 and 2 have tangency planes along their extreme transversal edges and the planes corresponding to each panel are parallel to each side of the timberfabric module. In other terms, $\Pi\left(A_{1}, \hat{\mathbf{n}}^{A_{1}}\right) \| \Pi\left(A_{2}, \hat{\mathbf{n}}^{A_{2}}\right)$ and $\Pi\left(B_{1}, \hat{\mathbf{n}}^{B_{1}}\right) \| \Pi\left(B_{2}, \hat{\mathbf{n}}^{B_{2}}\right)$.

- The four normal vectors ${ }^{A_{1}},{ }_{2},{ }^{B_{1}}$ and $B_{2}$ do not have a component along $\hat{\mathbf{e}}_{2}$ . That is $\hat{\mathbf{n}}^{M} \cdot \hat{\mathbf{e}}_{2}=0, M \in\left\{A_{1}, A_{2}, B_{1}\right.$, $\left.B_{2}\right\}$.

\section{Identification of Intermediate Geometric Parameters}

To quantitatively describe the geometry of the timberfabric module, four intermediate variables are introduced. Those variables are expressed as functions of components of the displacement and rotation vectors of particular points, so that they can be extracted from the numerical results of the FE model. A geometric interpretation of those variables is given in Fig. 6. More specifically,

$\alpha=\left|\theta_{2}^{M}\right|=\arccos \left(\hat{\mathbf{n}}^{M} \cdot \hat{\mathbf{e}}_{3}\right), \quad M \quad \in\left\{A_{1}\right.$, $\left.A_{2}, B_{1}, B_{2}\right\}$ is the absolute value of the inclination angle of the tangency planes at the extremities of a timberfabric module.

$\Theta=\left|\theta_{1}^{C_{1}}-\theta_{1}^{C_{2}}\right|=2\left|\theta_{1}^{C_{1}}\right|=2\left|\theta_{1}^{C_{2}}\right| \quad$ is the angle between the panels at mid-span.

$L_{f}=L+u_{1}^{B_{2}}$ and $h_{m}=u_{3}^{c_{1}}=u_{3}^{c_{2}}$ are respectively the span and the height of a timberfabric module.
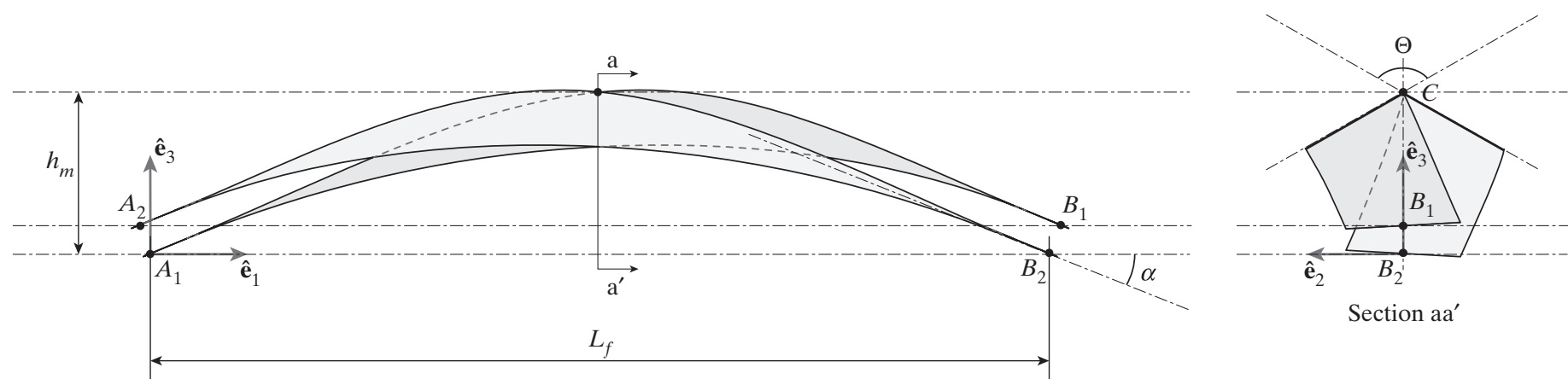

Fig. 6: Definition of "intermediate variables" 


\section{Parametric Study}

So far, the following has been introduced:

- The initial parameters, $L, W, t$ and $h_{c}$ to describe the geometry of the timber panels and the interaction condition at their ends.

- The homogenized mechanical properties of the panels: $E_{1}, E_{2}, v_{12}, G_{12}$.

- The intermediate variables $\Theta, \alpha, L_{f}$, $h_{m}$ and $\Theta$ to describe the geometry of the timberfabric module.

By adapting the script that calculates the deformed configuration of the panels of a timberfabric module, it is possible to run a parametric study to observe the influence of the geometric parameters and the mechanical properties on the intermediate variables that quantitatively describe the geometry of a TM.

The results of this study provide very useful information for the design of vaults with imposed dimensions. For example, if timberfabric modules of a given length rotation radius and material are required, the results of the study will help to determine the corresponding length and width of the panels.

To choose the size of the mesh used for this study, the authors first ran a parametric study on the number of elements per width of the panels. By accepting an error of $0,5 \%$ on the results of the intermediate variables, a mesh size corresponding to 12 elements per width of the panels, was chosen.

The most relevant output variables are $\alpha$ and $L_{f}$ as they will affect the geometry of the vault. The variables $h_{m}$ and $\Theta$ will rather affect the geometry of the connection pieces. For a given length of the panels, it was observed that $\alpha$ and $L_{f}$ are mostly influenced by the width $W$ as shown in Table 2. The thickness of the panels and height of the connector have a less critical influence. Figure 7 gives a graphical illustration of this influence.

\section{Parametric Model for Timber Vaults}

The tool presented in earlier section is further developed to enable the generation of a finite element model of timberfabric vaults. The script that builds the model uses a function of the FE program that enables one to use the result of a previous analysis as the initial state of a new analysis. Therefore the deformed configuration of the generated timberfabric module can be imported into a new model, copied, rotated and translated in the 3D space.

\begin{tabular}{|c|c|c|c|c|c|c|}
\hline$\frac{E_{2}}{E_{2}^{\text {ref }}}$ & $\frac{G_{I 2}}{G_{12}^{\text {ref }}}$ & $\frac{V_{12}}{V_{12}^{\text {ref }}}$ & $\alpha\left(^{\circ}\right)$ & $\frac{\boldsymbol{L}_{f}}{\boldsymbol{L}^{\text {reft }}}$ & $\Theta\left({ }^{\circ}\right)$ & $\frac{h_{m}}{L^{\text {ref }}}$ \\
\hline 0,8 & 1 & 1 & 40,33 & 0,948 & 125,89 & 0,1594 \\
\hline 0,9 & 1 & 1 & 40,31 & 0,948 & 125,93 & 0,1594 \\
\hline 1 & 1 & 1 & 40,29 & 0,948 & 125,96 & 0,1594 \\
\hline 1,1 & 1 & 1 & 40,28 & 0,948 & 125,98 & 0,1593 \\
\hline 1,2 & 1 & 1 & 40,27 & 0,948 & 126 & 0,1593 \\
\hline 1 & 0,8 & 1 & 39,17 & 0,950 & 124,90 & 0,1570 \\
\hline 1 & 0,9 & 1 & 39,74 & 0,949 & 125,44 & 0,1582 \\
\hline 1 & 1 & 1 & 40,29 & 0,948 & 125,96 & 0,1594 \\
\hline 1 & 1,1 & 1 & 40,83 & 0,947 & 126,46 & 0,1605 \\
\hline 1 & 1,2 & 1 & 41,36 & 0,946 & 126,93 & 0,1616 \\
\hline 1 & 1 & 0,8 & 40,46 & 0,948 & 125,99 & 0,1597 \\
\hline 1 & 1 & 0,9 & 40,38 & 0,948 & 125,98 & 0,1596 \\
\hline 1 & 1 & 1 & 40,29 & 0,948 & 125,96 & 0,1594 \\
\hline 1 & 1 & 1,1 & 40,20 & 0,948 & 125,93 & 0,1592 \\
\hline 1 & 1 & 1,2 & 40,11 & 0,949 & 125,89 & 0,1589 \\
\hline$\frac{W}{W^{\text {ref }}}$ & $\frac{h_{c}}{L_{c}^{\text {ref }}}$ & $\frac{t}{t^{\mathrm{ref}}}$ & $\alpha\left(^{\circ}\right)$ & $\frac{\boldsymbol{L}_{f}}{\boldsymbol{L}^{\text {ref }}}$ & $\theta\left(^{\circ}\right)$ & $\frac{h_{m}}{L^{\text {ref }}}$ \\
\hline 0,8 & 1 & 1 & 32,29 & 0,964 & 127,59 & 0,1333 \\
\hline 0,9 & 1 & 1 & 36,04 & 0,957 & 126,56 & 0,1460 \\
\hline 1 & 1 & 1 & 40,29 & 0,948 & 125,96 & 0,1594 \\
\hline 1,1 & 1 & 1 & 45,03 & 0,939 & 125,57 & 0,1732 \\
\hline 1,2 & 1 & 1 & 50,25 & 0,928 & 125,21 & 0,1873 \\
\hline 1 & 0.8 & 1 & 40,28 & 0,951 & 125,91 & 0,1564 \\
\hline 1 & 0.9 & 1 & 40,29 & 0,949 & 125,93 & 0,1579 \\
\hline 1 & 1 & 1 & 40,29 & 0,948 & 125,96 & 0,1594 \\
\hline 1 & 1.1 & 1 & 40,30 & 0,947 & 125,99 & 0,1609 \\
\hline 1 & 1.2 & 1 & 40,31 & 0,946 & 126,02 & 0,1624 \\
\hline 1 & 1 & 0,8 & 42,92 & 0,943 & 127,88 & 0,1651 \\
\hline 1 & 1 & 0,9 & 41,48 & 0,946 & 126,86 & 0,1620 \\
\hline 1 & 1 & 1 & 40,29 & 0,948 & 125,96 & 0,1594 \\
\hline 1 & 1 & 1,1 & 39,29 & 0,950 & 125,16 & 0,1571 \\
\hline 1 & 1 & 1,2 & 38,42 & 0,952 & 124,44 & 0,1552 \\
\hline
\end{tabular}

Table 2: Results of the parametrical study

The timberfabric modules of the new model can then be connected to one another and to the ground in order to form a statically equilibrated vault.

The geometric configuration of the vault is based on several assumptions and can be described by a new set of user-parameters. In this paper, only the family of vaults obtained by assembling identical TMs is studied.

The logic of the assembly and its implementation in a script are detailed in the following sections.

\section{Arch from Timberfabric Modules}

Let $\mathrm{TM} j$ be a timberfabric module. The notations introduced previously are extended by adding the number " $j$ " of the module in superscript. Accordingly, the local coordinates system associated to TM1 is written $\left(A_{1}^{1}, \hat{\mathbf{e}}_{1}^{1}, \hat{\mathbf{e}}_{2}^{1}, \hat{\mathbf{e}}_{3}^{1}\right.$,). For brevity, a unit vector at a given point $M$ of $\mathrm{TM} j$ will hereafter be denoted by $\hat{\mathbf{n}}_{M}^{j}$.
In the plane $\Pi\left(A_{1}^{1}, \widehat{\mathbf{e}}_{2}^{1}\right)$, there exits a unique circle that is tangential to the plane $\Pi\left(A_{1}^{1}, \hat{\mathbf{n}}_{A_{1}}^{1}\right)$ and passes through the points $A_{1}$ and $B_{2}$. By symmetry, the circle is also tangential to the plane $\Pi\left(B_{2}^{1}, \hat{\mathbf{n}}_{B_{2}}^{1}\right)$. From section "Geometrical Aspects of Timberfabric Modules" and according to the geometric construction of Fig. 8, the radius $R$ of this circle can be expressed as follows

$R=\frac{L_{f}}{2 \sin \alpha}$

In addition, the height $h_{2}$ of the arch $\left[A_{1}^{1} A_{2}^{1}\right]$ is defined by

$h_{2}=R \cdot(1-\cos \alpha)$

In the local coordinates system of TM1 the coordinates of the circle center $O^{1}$ are $\left(\frac{L_{f}}{2},-h_{1}, 0\right)^{T}$ with $h_{1}=R-h_{2}$.

Let us now introduce $(O, \hat{\mathbf{i}}, \hat{\mathbf{j}}, \hat{\mathbf{k}})$ the global Cartesian coordinates for representing the vault system. TM1 is placed in the global system such that 




Fig. 7: Graphic results of the parametric study for $t=8 \mathrm{~mm}$ and $h_{c}=80 \mathrm{~mm}\left(t / t^{\mathrm{ref}}=1, h_{c} / h_{c}{ }^{\mathrm{ref}}=1\right)$
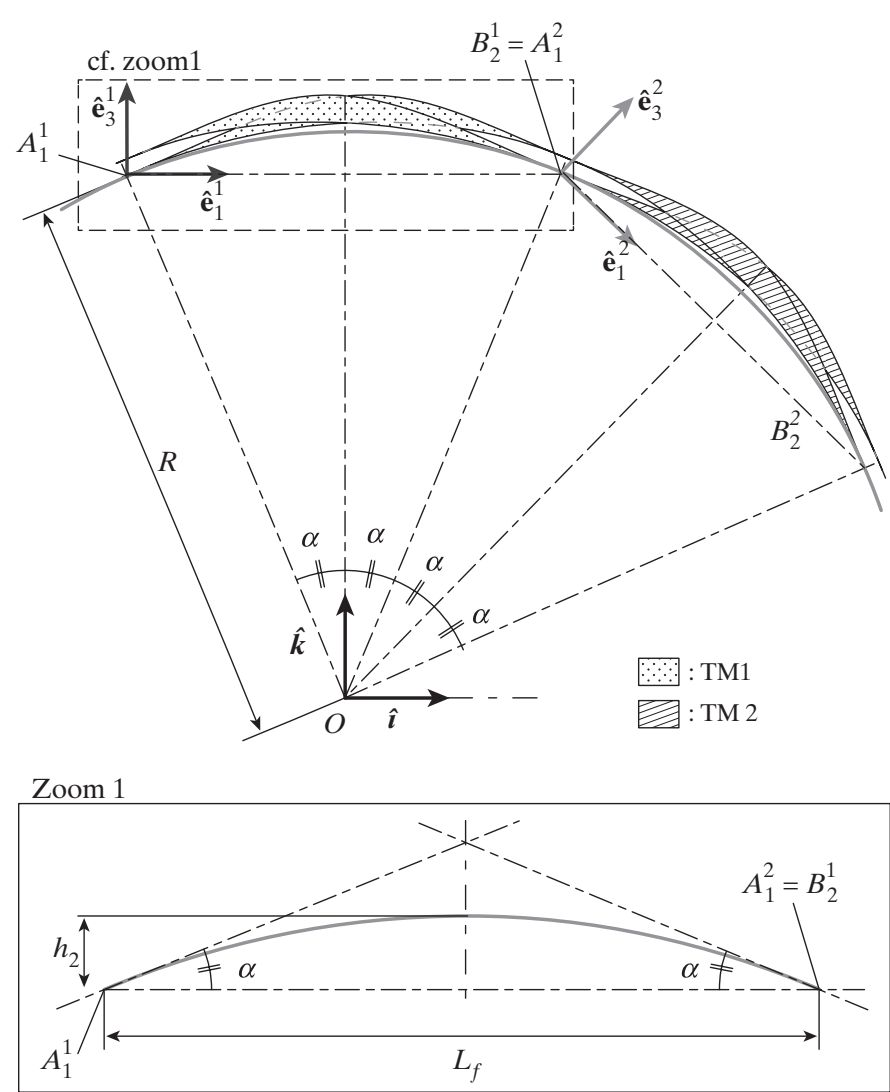

Fig. 8: Assembling timberfabric modules to form an arch
$O^{1}$ coincides with $O$ and $\hat{\mathbf{i}}, \hat{\mathbf{j}}$ and $\hat{\mathbf{k}}$ are respectively collinear to $\hat{\mathbf{e}}_{1}^{1}, \hat{\mathbf{e}}_{2}^{1}$ and $\hat{\mathbf{e}}_{3}^{1}$.TM2 is obtained by rotating TM1 clockwise about the axis $\hat{\mathbf{j}}$ by an angle $2 \alpha$, resulting in the following conditions at the ends of the modules

$B_{2}^{1}=A_{1}^{2}, B_{1}^{2}=A_{2}^{2}$,

$\hat{\mathbf{n}}_{B_{2}}^{1}=\hat{\mathbf{n}}_{A_{1}}^{2}, \quad \hat{\mathbf{n}}_{B_{1}}^{1}=\hat{\mathbf{n}}_{A_{2}}^{2}$

such that $\Pi\left(B_{2}^{1}, \hat{\mathbf{n}}_{B_{2}}^{1}\right)=\Pi\left(A_{1}^{2}, \hat{\mathbf{n}}_{A_{1}}^{2}\right)$ and $\Pi\left(B_{1}^{1}, \hat{\mathbf{n}}_{B_{1}}^{1}\right)=\Pi\left(A_{2}^{2}, \hat{\mathbf{n}}_{A_{2}}^{2}\right)$.

Equation (3) implies that there is a $C^{1}$-continuity between:

- "panel1" of TM1 and "panel2" of TM2

- "panel2" of TM1 and "panel1" of TM2

It was also observed that the boundary reaction force in $B_{2}^{1}$ and $B_{1}^{2}$ are respectively opposed to the boundary reaction force in $A_{1}^{2}$ and $A_{2}^{2}$. Those boundary reactions can therefore be replaced by interaction forces between TM1 and TM2. The nature of these interactions and their implementa- 
tion in the FE model will be detailed in section "Kinematical Interactions and Boundary Conditions". This twomodule arch is geometrically smooth and statically equilibrated.

Larger arches are obtained by assembling $n$ timberfabric modules in a way that reproduce the geometry that would be obtained with two continuous panels woven together $n$ times.

\section{Vaults from Arches}

In this paper, a cylindrical vault is typically obtained by juxtaposing several arches along the axis $(O, \hat{\mathbf{j}})$ of the global coordinate system. The distance between two arches is fixed by the parameter $s_{j}$ as explained below.

From one arch to the other, the modules are rotated by an angle $\alpha$ around the axis $(O, \hat{\mathbf{j}})$ as illustrated in Fig. 9. This implies that the extremity of an arch can either be a full timberfabric module or half of a timberfabric module.

Up to this point, describing kinematical relations between several nodes has simulated the interactions between the panels of a timberfabric module. In order to link the arches together, connection pieces are introduced in the model and referred to as "connectors".

Let TM1 be a timberfabric module placed in the global coordinates system as in section "Arch from Timberfabric Modules". As illustrated in Fig. 9, the timberfabric module TM2 is obtained by a translation of TM1 of vector $\mathbf{T}=s_{j} \hat{\mathbf{j}}$ and a rotation of angle $\alpha$ around the axis $(O, \hat{\mathbf{j}})$. The mid-surface of the connectors belongs to the plane $\Pi\left(C^{1}, \widehat{\mathbf{e}}_{1}^{1}\right)$ : this plane is perpendicular to the tangency planes $\Pi\left(A_{1}^{2}, \hat{\mathbf{n}}_{A 1}^{2}\right)$ and $\Pi\left(A_{2}^{2}, \hat{\mathbf{n}}_{A 2}^{2}\right)$ and includes the points $C^{1}$, $A_{1}^{2}$ and $A_{2}^{2}$.

Considering the sections of TM1 and TM2 by this plane, the geometry of the connectors is given by the points $\{\mathrm{a}, \mathrm{b}, \mathrm{c}, \mathrm{d}, \mathrm{e}, \mathrm{f}, \mathrm{g}, \mathrm{h}\}$. Given in Table 3 are the coordinates of these points in the system $\left(C^{\prime 1}, \widehat{\mathbf{e}}_{2}^{1}, \widehat{\mathbf{e}}_{3}^{1}\right)$ (see Fig. 9). The parameters introduced in Table 3 are given by

$l_{1}=\left(h_{m}-h_{2}\right) \cdot \tan \Theta / 2$

$l_{2}=l_{1}+h_{c} / \tan \Theta_{2}$

$h_{3}=h_{c} \cdot\left(1+\frac{1}{\tan \Theta / 2 \cdot \tan \Theta_{2}}\right), \Theta_{2}=\frac{\pi+\Theta}{4}$

Pieces of wood that are cut out of $25 \mathrm{~mm}$ thick laminated timber panels
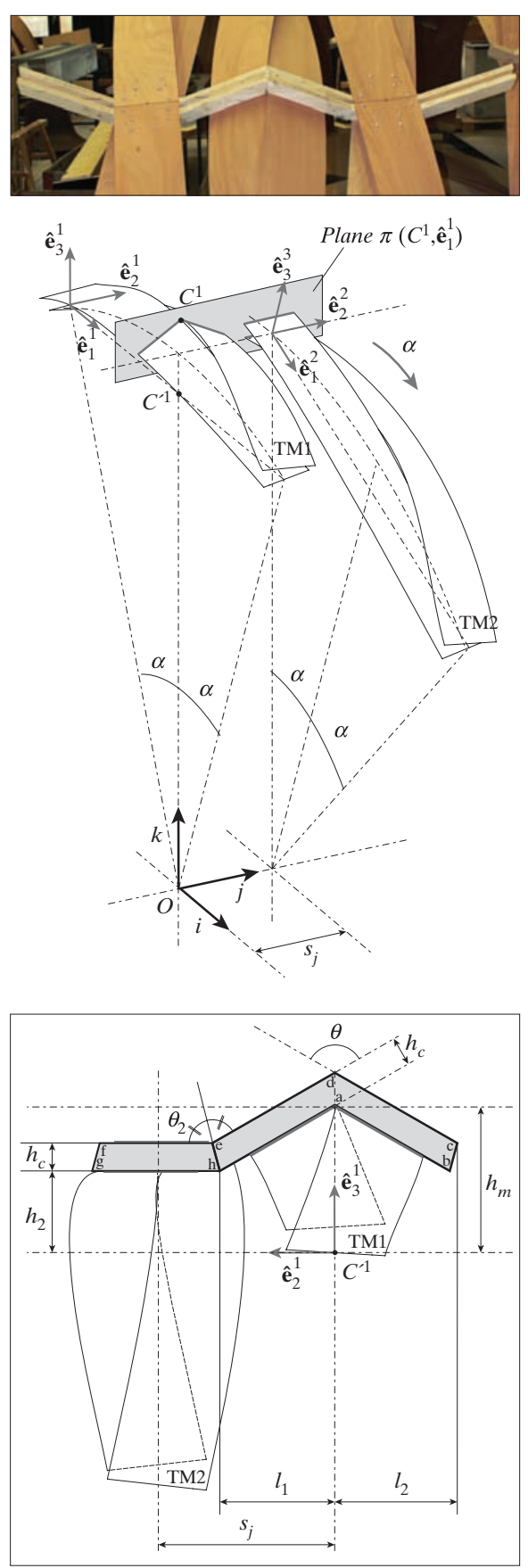

Fig. 9: Connecting arches together

(class of resistance C30) are assembled to make the connectors. The detailed construction of the connection pieces is not covered in this paper.

In the FE model, those connectors are modeled as two planar shells rigidly bonded along the edge [e,h]; "connector 1 " and "connector 2 " will refer respectively to the planar surfaces defined by the sets of points $\{e, f, g, h\}$ and $\{a, b, c, d, e, h\}$.

\section{Map of the Vault}

The $\alpha$-rotation from one arch to the other implies that some timberfabric modules have to be cut in half so that all the arches can be connected to the ground at the same level. The halfmodules can be seen in Fig. 1. In order to give a clear description of the structure, the vault will be generated by assembling only halves of timberfabric modules together. The continuity of the panels of the full timberfabric modules will be restored in the $\mathrm{FE}$ model by specifying appropriate inter-

As represented in Fig. 10, the bottom part of the timberfabric module is referred to as half-module $k=1$ and the top part as half-module $k=2$ : each module consists of two halves of panels and one connector. With this virtual cutting of the timberfabric modules, the vault can be described by a chesslike diagram.

When using the script, the user inputs a value to the parameters " $n b a$ " and " $n b m$ " to specify the number of arches and the number of modules (in the study of the prototype, $n b a=3$ and $n b m=6)$.

The nature of the half-module corresponding to a module $j$ of the arch $i$ is described by the variable $k$ for which possible values are 1 or 2 . The choice of the value of $k$ for $i=1$ and $j=1$, i.e. $k(1,1) \equiv k_{11}=1$ or 2 , determines the value of $k$ for any other integer couple $(i, j)$ as

$$
\begin{aligned}
& k(i, j)=\bmod \left(i+j+\left(k_{11}-1\right), 2\right)+1, \\
& 1 \leq i \leq n b a, 1 \leq j \leq n b m
\end{aligned}
$$

$\bmod (a, b)$ being the positive remainder in the division of $a$ by $b$, where $a$ and $b$ are positive integers.

\section{Geometric Assembly Process}

The geometric assembly is achieved by performing a double loop on $i$ in $[1,2, \ldots, n b a]$ and $j$ in $[1,2, \ldots, n b m]$. action conditions.

\begin{tabular}{|c|c|c|c|c|}
\hline Points & $\mathbf{a}$ & $\mathbf{b}$ & $\mathbf{c}$ & $\mathbf{d}$ \\
\hline Coordinates* & $\left(0, h_{m}\right)$ & $\left(-l_{1}, h_{2}\right)$ & $\left(-l_{1}, h_{2}+h_{c}\right)$ & $\left(0, h_{m}+h_{3}\right)$ \\
\hline Points & $\mathbf{e}$ & $\mathbf{f}$ & $\mathbf{g}$ & $\mathbf{h}$ \\
\hline Coordinates* $^{*}$ & $\left(l_{1}, h_{2}+h_{c}\right)$ & $\left(2 s_{j}-l_{2}, h_{2}+h_{c}\right)$ & $\left(2 s_{j}-l_{1}, h_{2}\right)$ & $\left(l_{1}, h_{2}\right)$ \\
\hline
\end{tabular}

*In $\left(C^{\prime 1} \widehat{\mathbf{e}}_{2}^{1}, \widehat{\mathbf{e}}_{3}^{1}\right)$

Table 3: Coordinates of the points defining the geometry of the connectors 


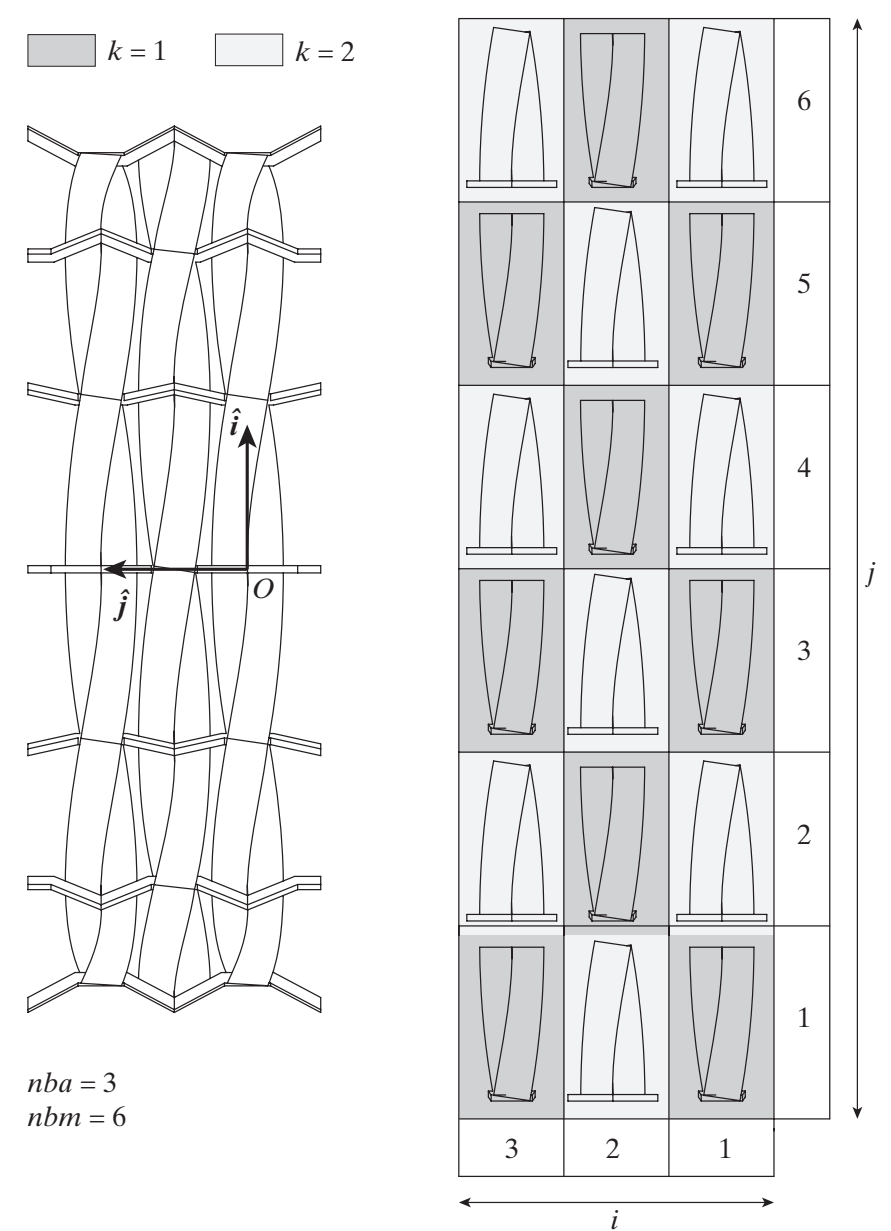

Fig. 10: "Map" of a timberfabric vault
For each couple $(i, j)$, the three parts corresponding to the half module $k(i, j)$ are imported into the model, then translated and rotated.

First, the instances of the parts are placed in the global coordinate system $(O, \hat{\mathbf{i}}, \hat{\mathbf{j}}, \hat{\mathbf{k}})$ in such a way that their reference point $A_{1}$ (also noted $A_{1}^{j} j=1, n b m$ in section "Arch from Timberfabric Modules") and point $O$ are coincident and local and global axes are parallel.

Then, they are placed in the vault system by applying the translation vector $\boldsymbol{T}$ and rotation angle $\beta$ about the global axis $\hat{\mathbf{j}}$ as defined below

$\mathbf{T}=-\frac{L_{f}}{2} \hat{\mathbf{i}}+(i-1) \cdot s_{j} \cdot \hat{\mathbf{j}}+\left(R-h_{2}\right) \cdot \hat{\mathbf{k}}$

$\beta=\left(j+k(i, j)-1-\frac{n b m}{2}\right) \cdot \alpha$

By taking $n b a=1$ (i.e. one arch) and $n b m=2$ (i.e. two modules) in Eq. (6), the positioning of the two modules TM1 and TM2 in the global coordinate system is recovered.

\section{Kinematical Interactions and Boundary Conditions}

Finally, to model the vault, it is necessary to introduce kinematical

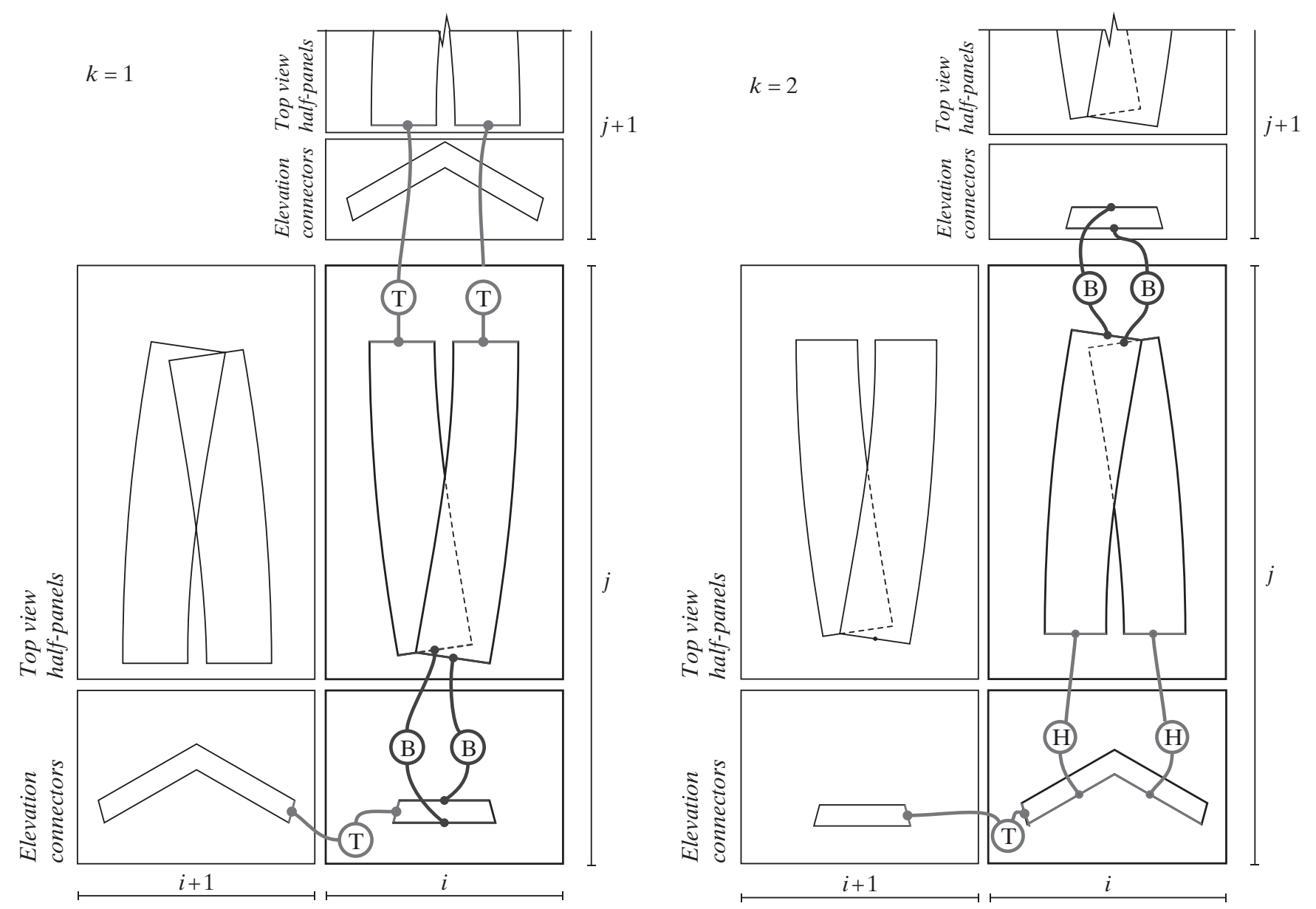

Fig. 11: Kinematical interactions between constitutive elements of the model 
interactions between the different geometric entities of the model in order to:

- restore the material continuity of the elements that have been split (this split was done to facilitate the description of the virtual assembly process)

- simulate the kinematic relations between the panels and the connectors.

The definition of those interactions is also generated automatically by looping over $i$ in $[1,2, \ldots, n b a]$ and $j$ in $[1,2, \ldots, n b m-1]$. Figure 11 summarizes the interactions that are defined depending on the value of $k(i, j)$ and the letters refer to the nature of the interaction between the two edges that are connected.

For all the elements that have been virtually "cut" in the model, the corresponding meshes require to be tied back together. In Fig. 11, the letter 'T' stands for "Tie": accordingly, the DOFs of each node of one edge are constrained to the DOFs of the corresponding node of the other edge.

For the interactions between the panels and the connectors, there are two objectives: ensuring the static equilibrium of the vault and imposing geometric conditions as described in section "Geometric Description". In practice, the panels are fixed on the connectors by ponctual screws with given characteristics and a given repartition. To model the global effect on the structure's behavior of those locally complex connections, all the nodes of an edge are constrained to the central node of this edge and simple kinematical interactions are defined between pairs of central nodes. Two different connections are implemented as represented in Fig. 11: "B" stands for "beam" and "H" stands dor "hinge". The beam constrains all the DOFs of one node to the DOFs of the connected node whereas the hinge constrains all the DOFs except the rotational DOF along the axis of the edge.

Finally, the boundary conditions consist in blocking all the DOFs of the connectors of the extremities (corresponding to the couples $(i, 1)$ and $(i$, $n b m+1)$ for $i$ in $[1,2, \ldots, n b a])$.

\section{Examples (application)}

As stated before, the parametric model is an interesting tool for modifying the architectural design of the initial structure. Figure 12 illustrates how very different geometry can be generated by varying the initial and intermediate
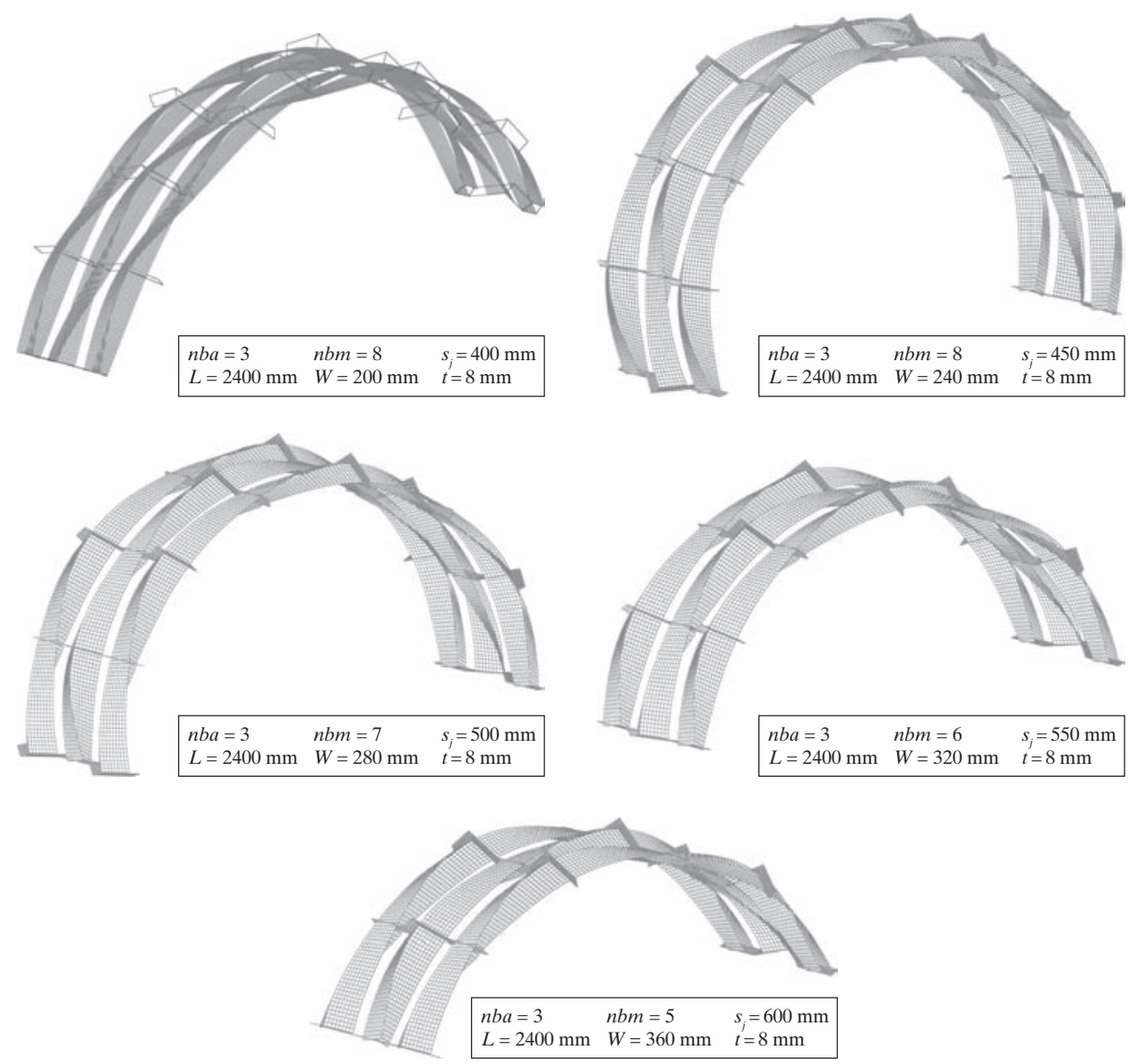

Fig. 12: Geometries generated for several values of the initial and intermediate parameters $\left(h_{c}=80 \mathrm{~mm}\right.$ and material properties are given in Table 1)

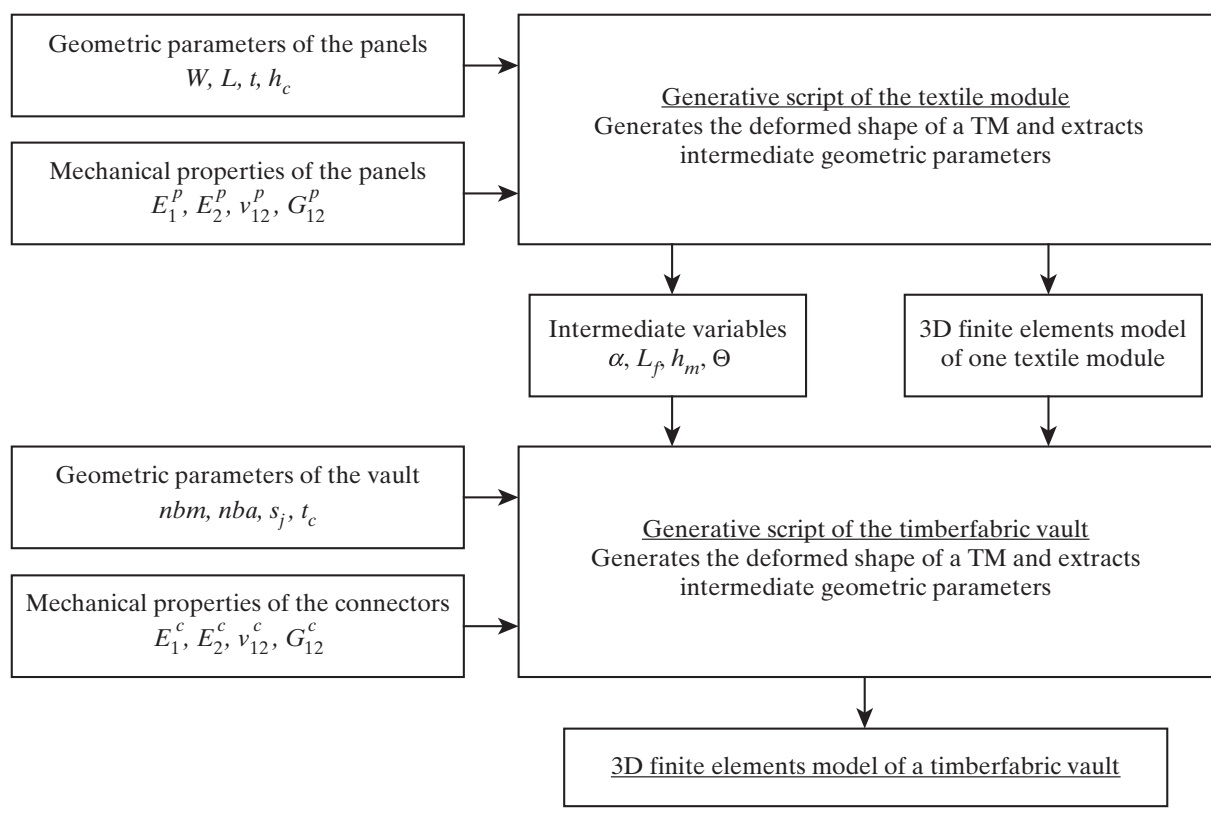

Fig. 13: Synthetic diagram of the complete script

parameters of the model. This will be of particular interest during the conception phase as architects, planners and engineers could discuss and jointly visualize the variation of geometric parameters such as the curvature radius as shown here. The visualisation of the global form is crucial for that interdisciplinary understanding necessary to achieve harmony of structural function.

\section{Conclusion}

The numeric tool described in this paper has the specificity of being useful for the architectural and the structural design of timberfabric structures. 
Indeed, the complexity of such structures resides essentially in the interdependence of the geometrical and mechanical aspects. The main challenge was therefore to find a way to describe quantitatively the geometry of one constitutive element (a TM) with a small set of variables and to elaborate a procedure to calculate them by generating the whole deformed geometry.

Rather than trying to reproduce the exact steps of deformation necessary to assemble flat panels into a timberfabric module and to assemble several TM together, the strategy was to reproduce directly the final geometry. The objectives came from the observation of the results of the empirical approach for the prototype shown in Fig. 1. This also means that the geometry of the TMs that had been calculated were not the geometry of self-equilibrated TMs but instead a geometry "extracted virtually" from the vault having more complex boundary conditions.

The illustration in Fig. 13 synthesizes the actions performed automatically by the scripts and highlights the input parameters that need to be specified by the user as well as the intermediate variables that need to be introduced in order to enable the automatic generation of the vault after having calculated one timberfabric module.

It is important to notice that this strategy of generation of the modules from flat panels allows one to calculate the stress state of the panels that constitute a timberfabric module: this information is essential to study the long-term behavior and the resistance of such structures.

All in all, the complexity of timberfabric structures calls for the elaboration of powerful tools. The work presented in this paper gives a strategy that generates FE models for a rather simple family of timberfabric vaults but the structural analysis still has to be performed manually. Further elaboration of the tool will allow dealing with structures made from timberfabric modules of various sizes and automatic verification of service and ultimate limit states.

\section{References}

[1] Garcia M (ed). Architextiles. Archit. Des. 2006; 76(6): 5-20.

[2] Tsukui N (ed). Cecil Balmond. A+U Special Issue, 2006.

[3] Weinand Y. Innovative timber constructions. J. Int. Assoc. Shell Spat. Struct. 2009; 50(161): 111-120.
[4] Hudert M. Timberfabric-Applying Textile Assembly Principles for Wood-Construction in Architecture. PhD Thesis, EPFL, Switzerland, 2013.

[5] Weinand Y, Hudert M. Timberfabric: applying textile principles on a building scale. Archit Des. 2010; 80(4): 102-107.

[6] Hudert M, Weinand Y. Structural timber fabric: applying textile principles on building scale ICSA2010 1st International Conference on Structures \& Architecture, Guimaraes, Portugal, 2010.

[7] Hudert M, Weinand Y. Timberfabric: innovative lightweight structures. IABSE-IASS Symposium, London, Great-Britain, 2011.

[8] Sistaninia M, Hudert M, Humbert L, Weinand Y. Experimental and numerical study on structural behavior of a single textile module. Engnr. Struct. 2013; 46: 557-568.

[9] ABAQUS Version 6.11. Documentation: Abaqus Scripting User's Manual and Reference Manual. Dassault Systems; 2011.

[10] Daniel, I, Ishai O. Engineering Mechanics of Composite Materials. Oxford University Press: Oxford, 1994

[11] Bodig J, Jayne BA. Mechanics of Wood and Wood Composites. Van Nostrand Reinhold Company, New York, 1982.

[12] Reddy, JN. An Introduction to Non-linear Finite Element Analysis. Oxford University Press: New York, 2004.

[13] Teboply Okoume plywood datasheet, http:// www.tebopano.com.

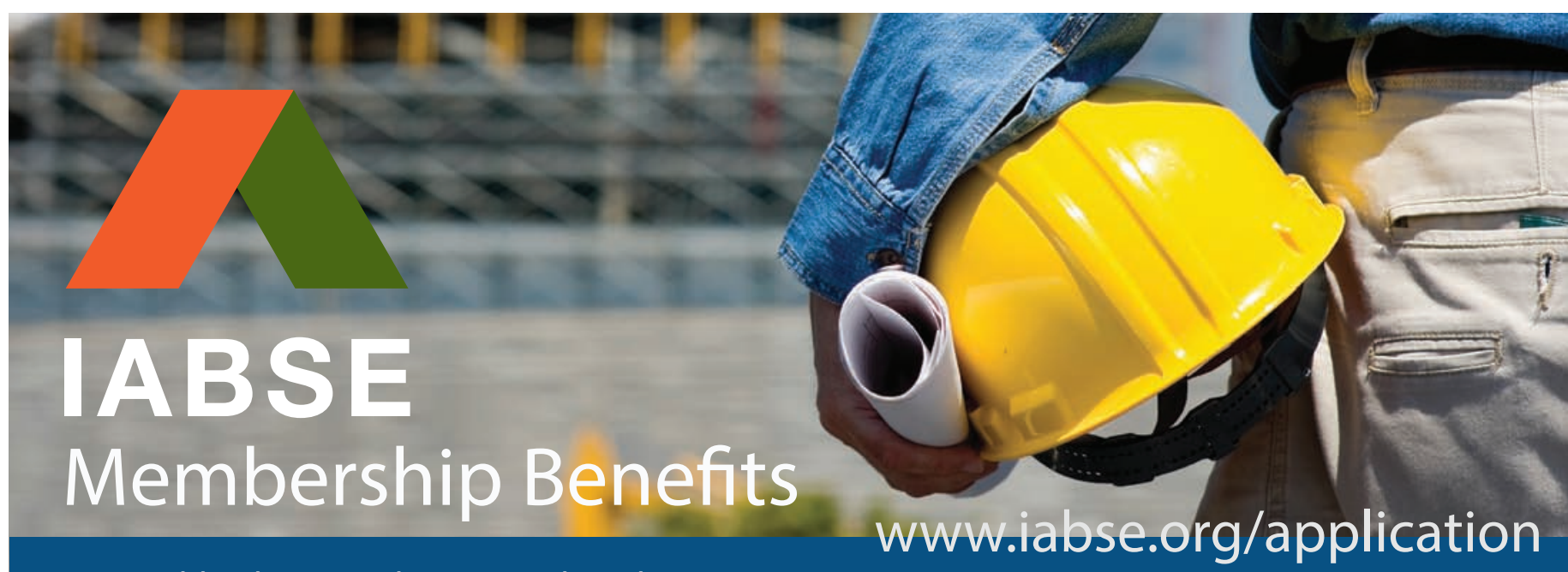

- Worldwide network in research and practice

- Free subscription to quarterly journal SEI

- Free access to electronic archive of SEl since 1991

- Free subscription to E-books (Structural Engineering Documents, SED)

- Reduced prices for IABSE Publications

- Reduced registration fees for IABSE Conferences

- Possibility to join Technical Groups

- Opportunity to join activities of National Groups

- Free Job Advertising on www.iabse.org

- Access to Members Area on www.iabse.org including Members Directory 